\title{
Moving knowledge into action for more effective practice, programmes and policy: protocol for a research programme on integrated knowledge translation
}

Ian D. Graham ${ }^{*}$, Anita Kothari ${ }^{2}$, Chris McCutcheon ${ }^{3}$ and On behalf of the Integrated Knowledge Translation Research Network Project Leads

\begin{abstract}
Background: Health research is conducted with the expectation that it advances knowledge and eventually translates into improved health systems and population health. However, research findings are often caught in the know-do gap: they are not acted upon in a timely way or not applied at all. Integrated knowledge translation (IKT) is advanced as a way to increase the relevance, applicability and impact of research. With IKT, knowledge users work with researchers throughout the research process, starting with identification of the research question. Knowledge users represent those who would be able to use research results to inform their decisions (e.g. clinicians, managers, policy makers, patients/families and others). Stakeholders are increasingly interested in the idea that IKT generates greater and faster societal impact. Stakeholders are all those who are interested in the use of research results but may not necessarily use them for their own decision-making (e.g. governments, funders, researchers, health system managers and policy makers, patients and clinicians). Although IKT is broadly accepted, the actual research supporting it is limited and there is uncertainty about how best to conduct and support IKT. This paper presents a protocol for a programme of research testing the assumption that engaging the users of research in phases of its production leads to (a) greater appreciation of and capacity to use research; (b) the production of more relevant, useful and applicable research that results in greater impact; and (c) conditions under which it is more likely that research results will influence policy, managerial and clinical decision-making.
\end{abstract}

Methods: The research programme will adopt an interdisciplinary, international, cross-sector approach, using multiple and mixed methods to reflect the complex and social nature of research partnerships. We will use ongoing and future natural IKT experiments as multiple cases to study IKT in depth, and we will take advantage of the team's existing relationships with provincial, national and international organizations. Case studies will be retrospective and prospective, and the 7-year grant period will enable longitudinal studies. The initiation of partnerships, funding processes, the research lifecycle and then outcomes/impacts post project will be studied in real time. These living laboratories will also allow testing of strategies to improve the efficiency and effectiveness of the IKT approach.

(Continued on next page)

\footnotetext{
* Correspondence: igraham@ohri.ca

${ }^{1}$ School of Epidemiology and Public Health, University of Ottawa, 307D- 600

Peter Morand Crescent, Ottawa, ON K1G 5Z3, Canada

Full list of author information is available at the end of the article
} 
(Continued from previous page)

Discussion: This is the first interdisciplinary, systematic and programmatic research study on IKT. The research will provide scientific evidence on how to reliably and validly measure collaborative research partnerships and their impacts. The proposed research will build the science base for IKT, assess its relationship with research use and identify best practices and appropriate conditions for conducting IKT to achieve the greatest impact. It will also train and mentor the next generation of IKT researchers.

Keywords: Integrated knowledge translation, Knowledge translation, Implementation, Collaborative research, Research co-production, Knowledge mobilization, Research use, Participatory research, Knowledge transfer

\section{Background}

Health research is conducted with the expectation that it advances knowledge and eventually translates into improved health systems and population health. However, research findings are often caught in the know-do gap: they are not acted upon in a timely way or not applied at all. The failure to put research findings into action is therefore a major societal issue and contributes to the estimated \$200B (USD) of wasted research funding because the full potential of research was not realized [1].

The magnitude of the know-do gap has stimulated governments and research funders around the globe to recognize the importance of the active translation of research into action [2]. Where historically the problem of research underutilization was considered simply a dissemination failure (knowledge users unaware of research), some now suggest this gap results from knowledge production failures (not producing research addressing knowledge user problems).

A widely recognized and accepted tenet of knowledge translation is the integration of knowledge users throughout the research process. Integrated knowledge translation (IKT) is advanced as a way to increase the relevance, applicability and impact of results [3, 4]. It shares common principles with many collaborative research approaches: co-production of knowledge, participatory research, linkage and exchange, Mode 2 knowledge production, engaged scholarship and community-based participatory research [5-10].

This approach proposes researcher/knowledge user collaboration as a key step in achieving societal impact and a way for society to speak to science. IKT shifts from a paradigm where the researcher is expert to one where researchers and knowledge users are both experts bringing complementary knowledge and skills to the team. They collaborate on issue-driven research with the expectation the research will generate implementable solutions to long-standing problems [11]. With IKT, the knowledge users work with researchers throughout the research process, starting with identification of the research question-they are actively engaged in the governance, priority setting and conduct of the research. Knowledge users represent all those who would be able to use research results to inform their decisions (clinicians, managers, policy makers, patients/families and others). Increasingly, stakeholders (governments, funders, researchers, health system managers and policy makers, patients and clinicians) are showing interest in the idea that IKT generates greater and faster societal impact. Stakeholders include all those with an interest in the issue or research, some of whom (knowledge users) are in a position to make direct use of the research in decision-making while other stakeholders are not but nevertheless want the issues and problems addressed.

Research funders have also been considering how to increase the impact of the research that they fund and their role in knowledge translation [2, 12-14]. 'Integrated knowledge translation' is a Canadian research funder innovation, initially advanced by the Canadian Health Services Research Foundation [6] and referred to as Knowledge Exchange in the late 1990s/early 2000s. The concept was adopted and refined at the Canadian Institutes of Health Research which coined the term integrated knowledge translation in the first decade of 2000s [15]. To promote the concept of partnered research, these organizations created funding opportunities that required collaboration between researchers and knowledge users. The Canadian Institutes of Health Research launched several funding initiatives promoting IKT (e.g. Partnerships for Health System Improvement, Knowledge Synthesis, Knowledge to Action, Community Based Research on HIV/IADs, Industry-Partnered Collaborative Research funding opportunities $[16,17]$ and Canadian Institutes of Health Research's (CIHR's) Strategy for Patient-Oriented Research competitions) [18]. Alberta Innovates (formerly Alberta Innovates: Health Solutions), a provincial research funding agency, initiated Partnership for Research and Health Innovation in the Health System and the Collaborative Research and Innovation Opportunities to encourage collaboration between researchers and knowledge users and to help translate research into improved health [19]. Similar funding competitions exist globally (of note is the Patient-Centered Outcomes Research Institute in the USA that only funds collaborative research) [20]. Funders have also created centres mandated to promote 
knowledge user engagement, accelerate research application and more efficiently harvest research benefits (e.g. Australian Academic Health Centres, Dutch Academic Collaborative Centres, UK Academic Health Science Centres, UK Collaborations for Leadership in Applied Health Research and Care-CLAHRC) [21-25]. Interest in this concept has also been demonstrated recently by publication of papers and commentaries on the topic in at least one journal [3, 26-31].

Although IKT is broadly accepted, the actual research supporting it is limited and there is uncertainty about how best to conduct and support IKT. A limited number of scoping, realist and other reviews suggest there is value of researchers and knowledge users working collaboratively and others are underway [32-35]. Emerging scholarship focusing on participatory action research [8, 36], the UK CLAHRCs [37-42] and CIHR's evaluation of its IKT programmes [43, 44], is beginning to support the claims that IKT may lead to increased knowledge user capacity to use research; produce research that is more useful to knowledge users; increase the use of research in practice, health systems and policy decisions; and improve patient and health system outcomes. Studies are appearing describing how research partnerships work [45-47]. There is some evidence to suggest that in these collaborative research partnerships, researchers are the ones who benefit more by learning about the knowledge users' context $[11,48]$. Other studies reveal engagement of knowledge users can influence researchers' approaches to research and the review of grants $[49,50]$. However, the evidence is not yet conclusive on the impacts of IKT. At least one survey study failed to find an association between researcher/knowledge user engagement and research utility [51], suggesting that the factors determining effective IKT have yet to be clearly identified. Knowledge of IKT among researchers varies [52], and there is limited evidence about how researchers and knowledge users should go about collaborating. Despite the slim evidence base, stakeholder enthusiasm for IKT continues to grow. The expectation of enhanced impacts from IKT has seldom been critically assessed nor has the research partnering process been systematically studied. In response, Gagliardi and colleagues have recently suggested a research agenda for IKT [53].

The proposed research will build the science base for IKT, determine its effectiveness at increasing research use and identify best practices and appropriate conditions for conducting IKT to achieve the greatest impact on research use. The goals, objectives and outputs of the 7 -year research programme are described in Table 1.

\section{Conceptual framework guiding the research programme}

This research programme is informed by four main conceptual frameworks: (a) the Rycroft-Malone et al.
Table 1 Research programme goals, objectives and outputs

Goal 1: advance understanding of the concept of IKT from the perspectives of knowledge users, researchers, funders and universities

Objectives:

1. Describe researcher and knowledge user partnerships and the conditions under which these partnerships succeed or fail.

2. Identify research funding mechanisms designed to support IKT and explore their effect on knowledge user engagement in research.

3. Identify how university (dis)incentives influence researcher involvement in IKT.

Potential outputs: The knowledge generated will be immediately relevant to four groups: knowledge users and their organizations needing more relevant research, researchers wanting to do IKT, universities wanting to encourage IKT by faculty and funders wishing to make informed decisions about their policies and investments in support of IKT. The cumulative knowledge generated will fundamentally enhance our understanding of how and why researcher/knowledge user collaborations work and provide information on how to maximize the use of IKT as a strategy to address the underutilization of research.

Goal 2: assess the impacts of IKT

Objectives:

1. Synthesize existing research on the benefits and challenges of IKT.

2. Determine what ways and under what conditions IKT adds value to research findings for knowledge users.

3. Generate new evidence on the outcomes and impacts of IKT.

4. Meta-synthesize the findings from all our research programme projects to identify the benefits, risks and implications of doing IKT and the circumstances under which IKT is most appropriate and impactful.

Potential outputs: enhanced knowledge of the benefits, impacts, disadvantages and implications of IKT.

Goal 3: develop and adapt IKT theories and measurement tools Objectives:

1. Continue to evolve and test KT/implementation frameworks, particularly those incorporating IKT.

2. Develop and validate measures to assess partnering processes and impacts of IKT

3. Develop and test theory-based strategies/interventions designed to (a) support organizations' meaningful engagement with researchers and use of research and (b) strengthen knowledge user organizational leadership for IKT.

Potential outputs: further refinement of existing frameworks of IKT. Measures to reliably and validly assess IKT partnerships and their impacts. Better quantification of these phenomena for research purposes. Better research partnerships when partners use the measures to diagnose the quality of their relationships and identify challenges/ issues that they can address to prevent relationship breakdown.

Goal 4: convert evidence gained from the research programme into accessible resources and build IKT research capacity to accelerate capturing the benefits of health research in Canada and abroad Objectives:

1. Develop IKT training manuals, tools, sessions for researchers and knowledge users.

2. Increase capacity for IKT among researchers and knowledge users.

3. Engage funding agencies, universities, healthcare organizations to develop, implement and evaluate strategies to support researcher/ knowledge user engagement.

Potential outputs: guidance documents/manuals and training sessions for both knowledge users and researchers on how to develop and nurture effective research partnerships. Researchers skilled in IKT (10-14 new HQP, > 100 trainees). Knowledge users at multiple levels (executives, managers, policy makers, clinicians) capable of participating in and using research. Effective strategies to promote IKT. A strengthened research community of practice on IKT. Academic incentives and funder policies better aligned to support IKT. 
framework for collaborative research (FCR) [38, 42], (b) the Research Impact Continuum (RIC) translational framework [54], (c) the Knowledge to Action framework (KTA) for implementation [55, 56] and (d) the Gifford model of leadership [57, 58].

The FCR identifies nine domains influencing knowledge use and impact stemming from researcher/knowledge user collaborations: knowledge and knowledge production, facilitation, patient and public involvement, knowledge sharing and exchange, geography, actors/ agents, temporality, architecture of the knowledge user organization and its processes and context (the interconnecting and supporting relationships between all these domains). The RIC distinguishes between research and the practice of translation, highlights the role of research in translation, including IKT, and focuses attention on research impact. Indicators of success/impact guided by the RIC framework [54] include advances in knowledge (e.g. discoveries, publications), capacity enhancement (e.g. new HQP, trainees, researchers, knowledge users with IKT skills), health system and policy impacts (e.g. use of programme findings in decision-making). The KTA framework highlights the interplay between knowledge creation and application and identifies key components required for planned action. The Gifford leadership framework specifies the leadership and management behaviors that positively influence knowledge translation, including relation-oriented behaviors (supporting, developing, recognising others), change-oriented behaviors (visioning, providing direction, building coalitions) and task-oriented behaviors (clarifying roles, monitoring and procuring resources).

\section{Methods/design}

The approach to this Canadian Institutes of Health Research 7-year foundation grant is interdisciplinary and cross-sectoral, using multiple and mixed methods that best reflect the complex and social nature of research partnerships. Knowledge users are full members on the research programme and individual project teams and will continue to be actively involved in every step of the research process. To allow the research programme to be more inclusive than those listed on this proposal, the programme is organized as a researcher/knowledge user network known as the Integrated Knowledge Translation Research Network: Doing Research with the People Who Use it (https://iktrn.ohri.ca).The network has been specifically designed to include researchers interested in IKT who include early career, mid-career and senior researchers (referred to as IKT experts, currently $n=40$ ); IKT trainees (currently $n=16$ ), knowledge user experts from research funding agencies; charities; health services and health authorities and other organizations (currently $n=31$ ); and a methods resource group comprising knowledge translation and implementation science experts (currently $n=11$ ). When feasible to do so, we will use an IKT approach within the projects to expand the team's experiential knowledge of IKT mechanisms. All projects are guided by programme goals and objectives. Table 2 presents the programme work streams along with their objectives, rationale, research questions, level of partnerships and outputs.

Several knowledge syntheses are proposed to increase understanding of the concept of IKT (projects 1a-b), how IKT works and with what impact (projects $1 \mathrm{c}-\mathrm{d}$ ) and to identify tools to evaluate the partnering process (project 1e). A novel aspect of the research is that three initial multiple-case studies (projects $2 \mathrm{a}-\mathrm{c}$ ) anchor the programme during the first half of the grant. The case studies are both retrospective and prospective and will provide data and knowledge on how IKT works, its impact and the degree of engagement required to optimise impact. The case studies will provide insight into IKT partnerships at two levels:

(a) Inter-organizational: partnerships between BORN (Better Outcomes Registry and Network) Ontario and hospitals providing maternity care (project $2 \mathrm{~b}$ );

(b) Regional: partnerships between Deakin University Centre for Quality and Patient Safety Research and health services in the State of Victoria, Australia (project 2a); university and regional health authority partnership (UNBC and Northern Health) (project 2c).

The case studies will be complemented with other projects focusing on other aspects of IKT and other programme objectives. For example, project $3 \mathrm{a}$ is intended to capture the network members' and organizations' experiential knowledge about working in an IKT way while project $3 \mathrm{~b}$ is about network members reflecting on the field and identifying where the science should focus. Several studies focus on funder programmes to promote research undertaken using an IKT approach (projects $4 \mathrm{a}-\mathrm{d}$ ). Other studies focus on the perspective of an organization that becomes the partner in an IKT project (projects $5 \mathrm{a}-\mathrm{b}$ ) or the perspective of the researcher or university (projects $6 \mathrm{a}-\mathrm{b}$ ). Project 7 is designed to develop and test an IKT questionnaire. Finally, projects $8 \mathrm{a}-\mathrm{d}$ are about IKT tools and developing training modules for researchers and knowledge users. We anticipate that research questions generated from projects will subsequently be embedded into future case studies as this will be an efficient way to study these topics without having to launch new stand-alone studies.

More case studies will be added as the grant proceeds. Several knowledge user partners are already identifying opportunities to study IKT 'in the field', and the project 


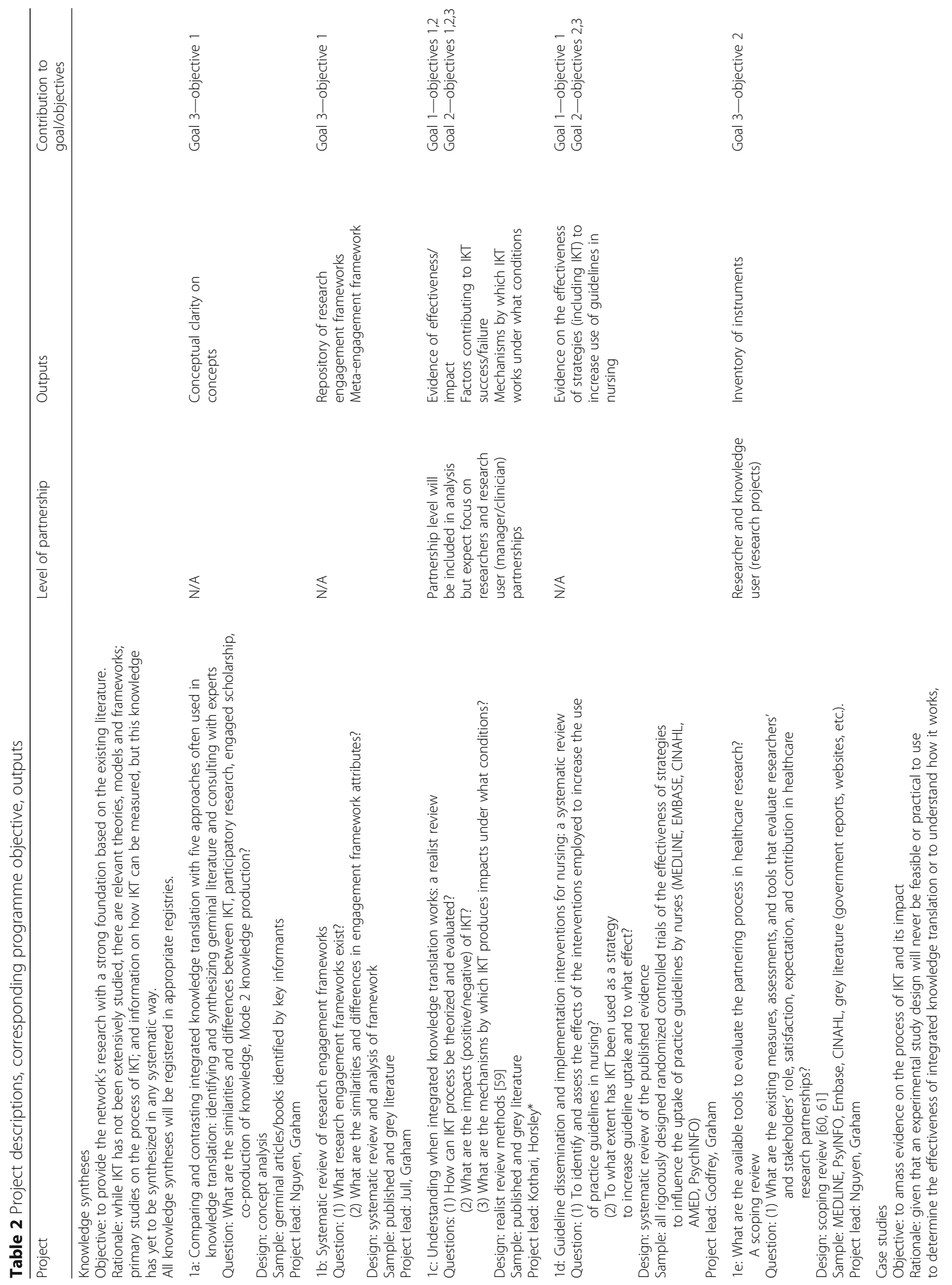




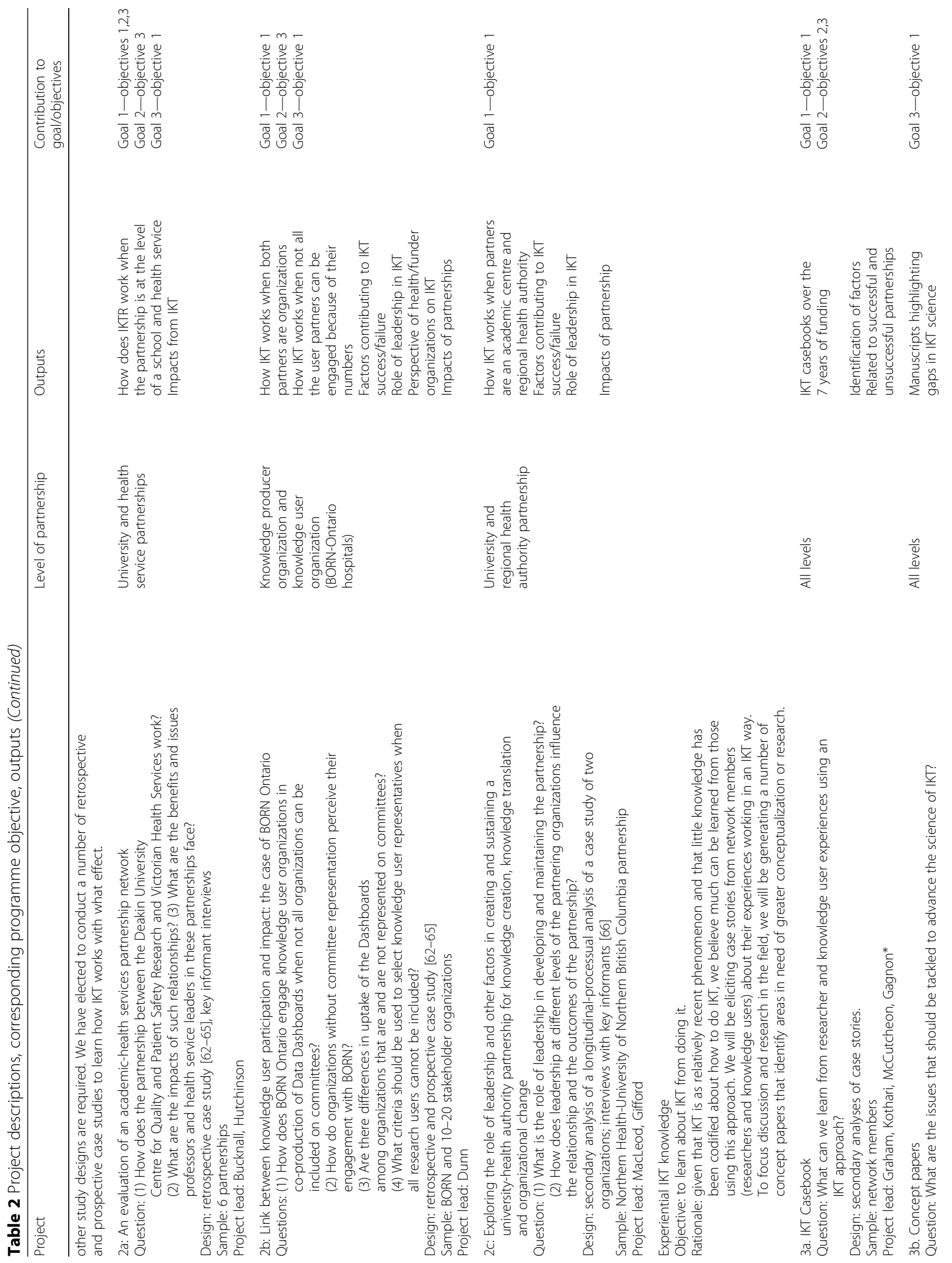




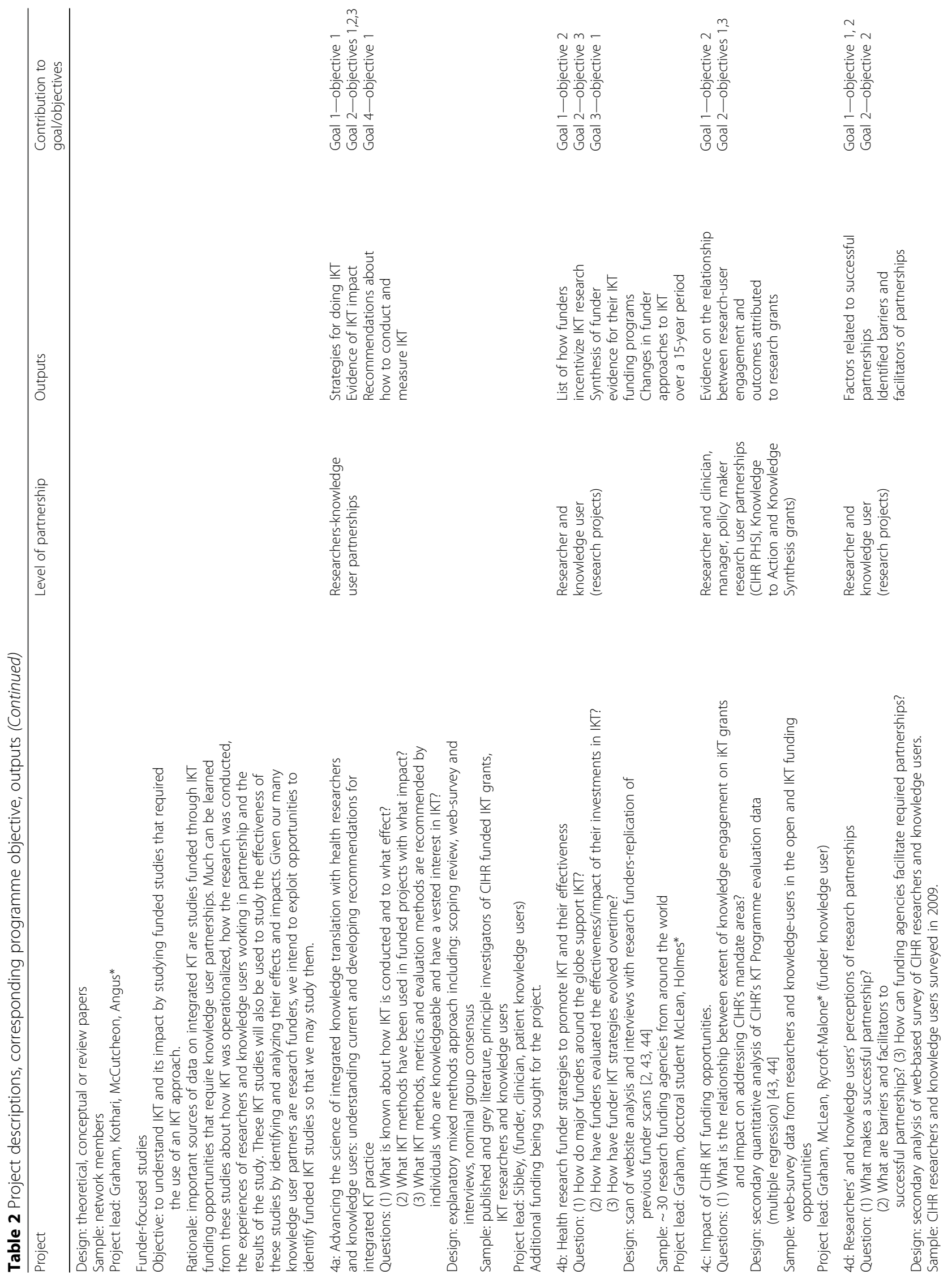




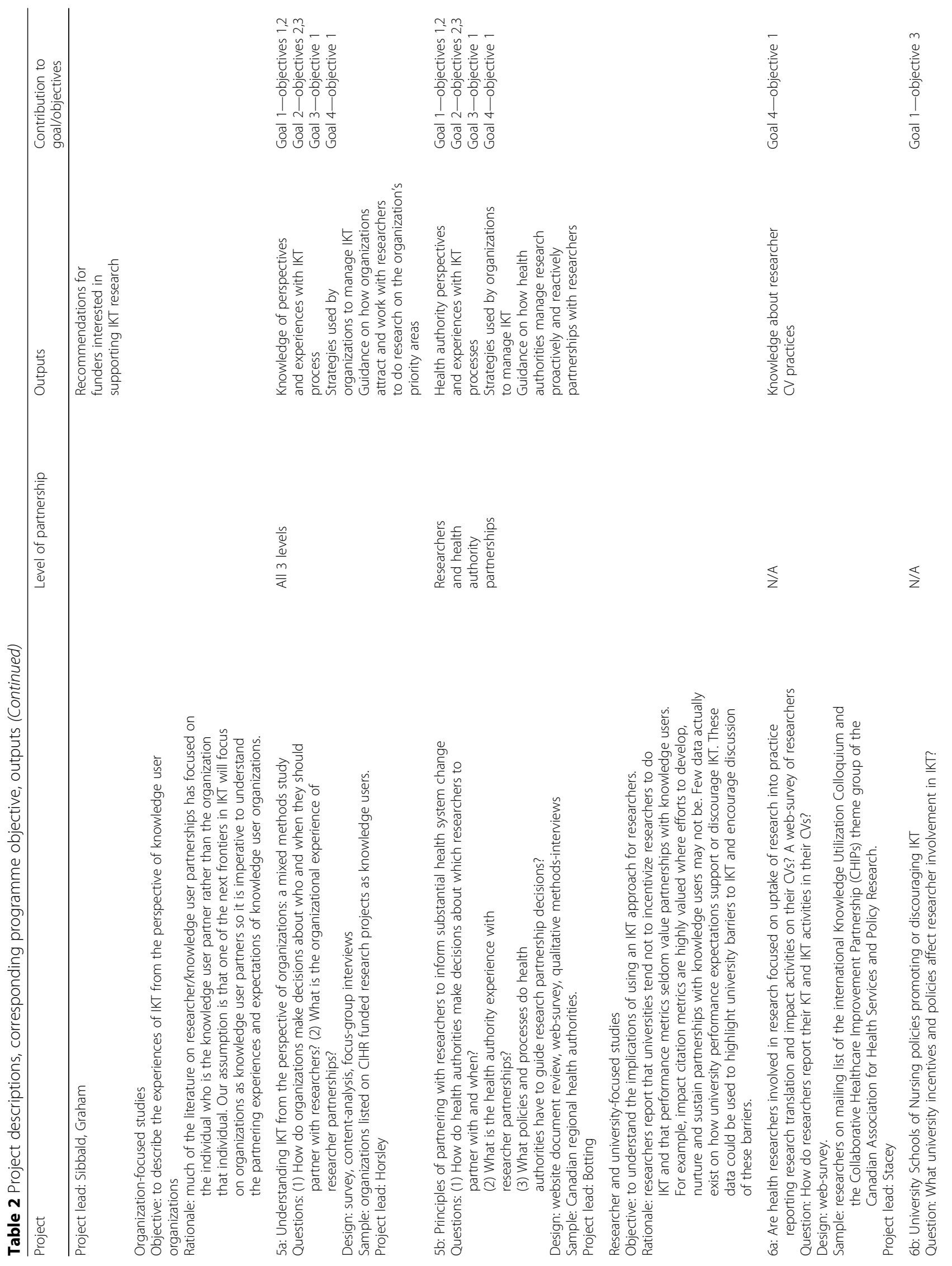




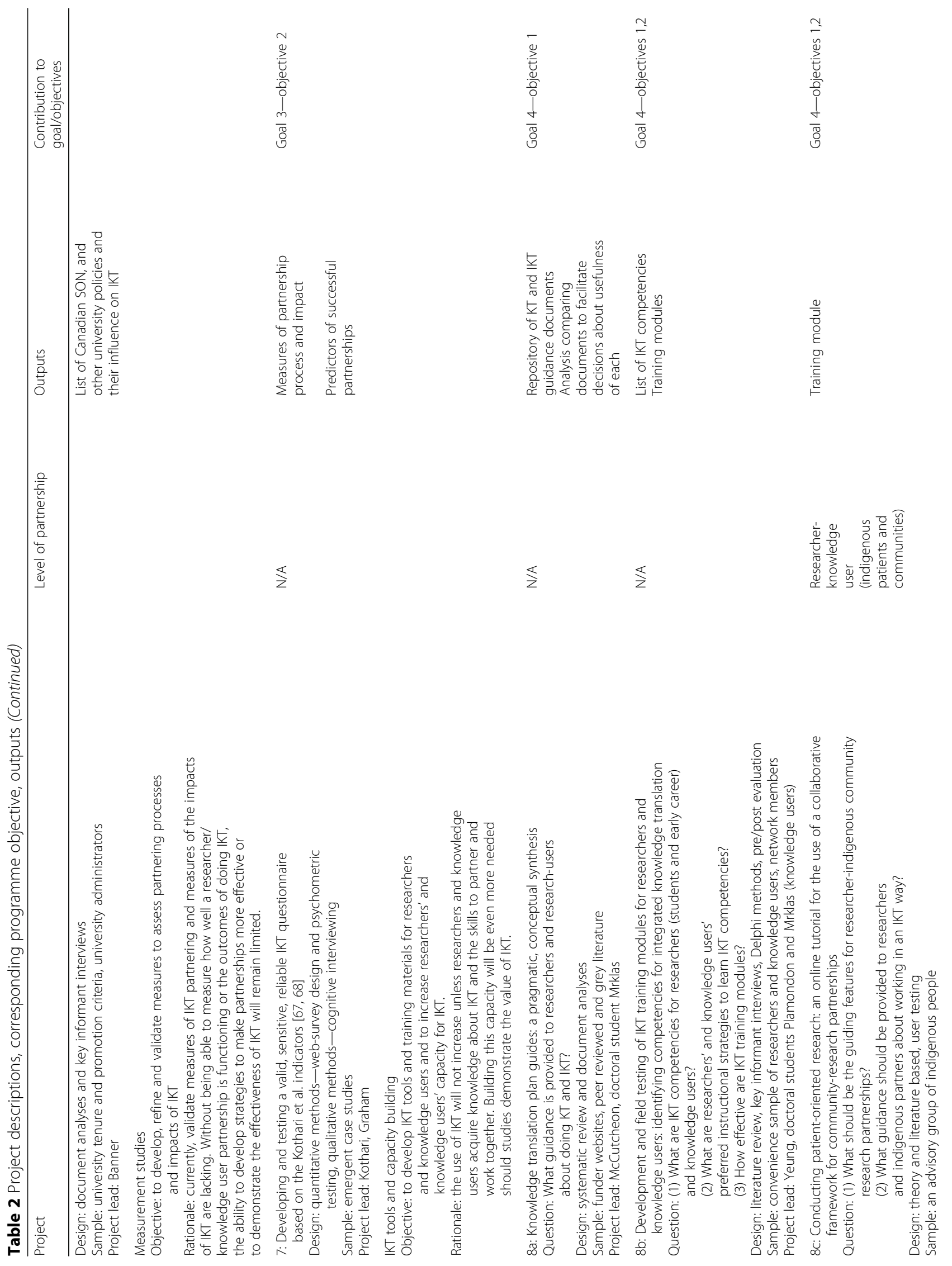




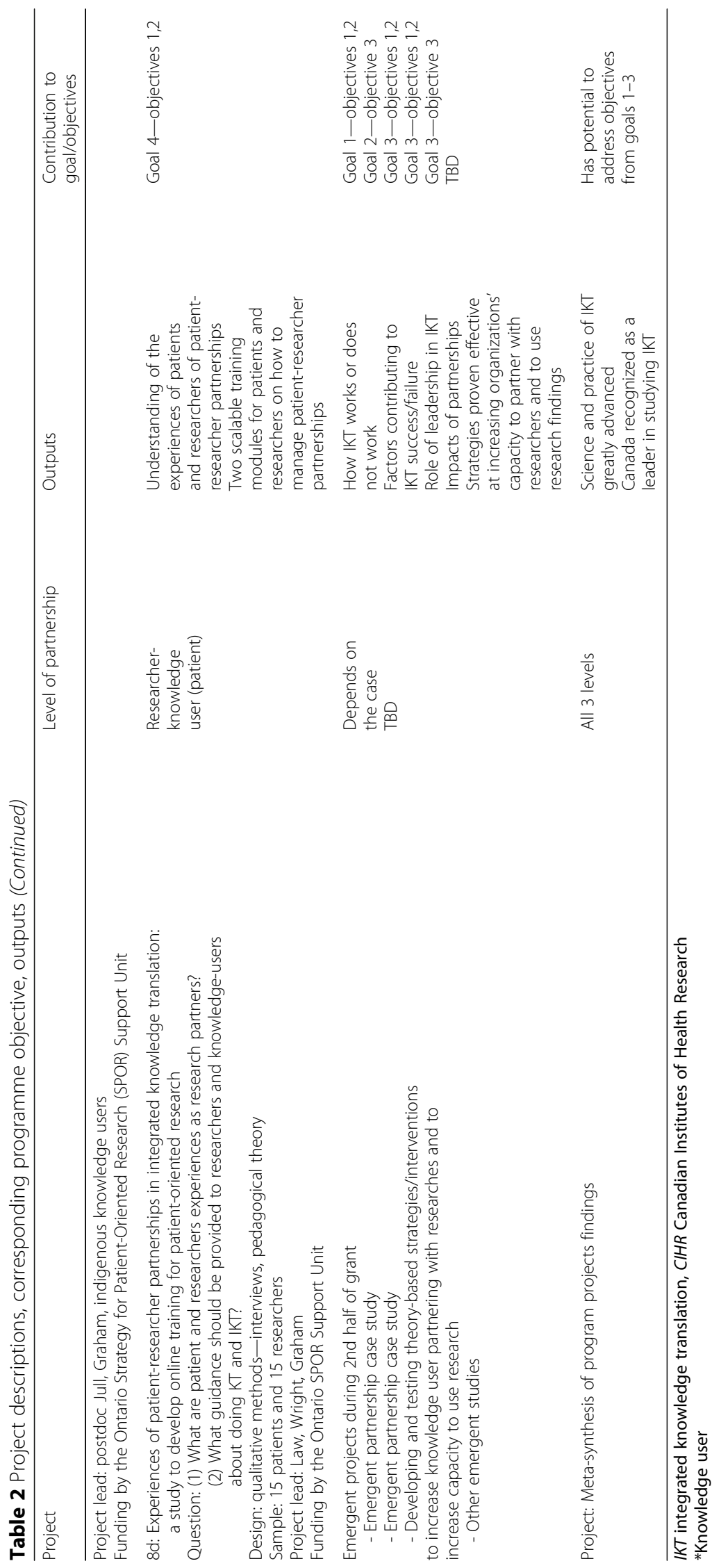


structure enables timely incorporation of these opportunities into the programme. In years $2-3$ of the grant, future case studies will be initiated. Initial criteria for selecting new projects will include addressing knowledge gaps identified in the programme's ongoing studies, prioritization of future studies by knowledge users and the Advisory Committee and feasibility. Towards year 4, intervention studies will be launched to test theorybased strategies to improve research partnering and build health organization capacity for research partnering and research use.

Finally, a meta-synthesis of findings from all projects will be completed to discern patterns and differences between different knowledge user groups (patients, clinicians, managers, policy makers), organizations (healthcare delivery institutions, health authorities, ministries of health, health research funders) and contexts and to develop materials to facilitate IKT and uptake of the findings. Team members are very interested in executives/managers, who have great potential to activate organizational change for research-informed decision-making but are understudied.

\section{Training}

Objective 2 of goal 4 is about creating a training environment for IKT research and supervising and mentoring graduate students and postdoctoral trainees and colleagues. To achieve this objective, the programme has an innovative and bold plan. We have incorporated funding to support one postdoc, two $\mathrm{PhD}$ and two master's students a year. This will produce five to seven master's, two to three PhDs and three to four postdocs over the life of the grant with expertise in the science and art of IKT. The CIHR KT evaluation [44] revealed that IKT projects are more likely to develop more highly qualified personnel per $\$ 100 \mathrm{k}$ grant than a grant of the same value in the open competition ([15], Table $2 \mathrm{p} 6$ ). Given the value of producing the next generation of KT scholars, we have also included student/trainee stipends to facilitate the involvement of students in as many of our projects and professional networks as possible. Over the life of the grant, this represents 35-40 studentships. We will also develop short internet training modules on various aspects of IKT for researchers and knowledge users.

The programme will also fund one to two researcher/ knowledge user internships/year (eight over the course of the grant). These will be for graduate students and postdoctoral trainees to spend 3 months sharing their research expertise with one of our knowledge user organization partners while they learn about policy making in the real world. This is an efficient way for trainees to learn about policy making while at the same time exposing the organizations to researchers in training. The internship programme will be modeled after CIHR's Science-Policy Fellowships developed by IDG when he was at CIHR (https://www.canada.ca/en/health-canada/ services/science-research/career-resources/fellowship-programs/science-policy-fellowships-program.html, Accessed 22 Dec 2017). Interns will be assigned both an academic and policy maker mentor.

\section{Governance and strategies to reduce risk to the research programme}

A governance structure is in place to ensure an enduring focus on excellence, flexibility and ability to capitalize on emerging opportunities and help the programme remain on track. An Executive Committee, chaired by a Program Leader (Scientific Director-IDG), will be responsible for day-to-day operations. It will include the Deputy Scientific Director (AK), two researchers, two knowledge users, one trainee and one research associate. Sub-committees responsible for science (IKT theory, methods and measures), impact (network performance monitoring) and training will provide leadership in these areas. The impact committee will convene an impact workshop with the project leaders to produce a logic model or theory of change for the network and determine how to collect data to test it. An international Advisory Committee (AC) comprising knowledge users and IKT experts will provide guidance on all aspects of the programme, annually review the performance of programme projects and suggest strategies to reduce risk of bias in study design, data analysis and interpretation. Terms of reference for all committees will be finalized in collaboration with members and reviewed annually. The research programme team will use a collaborative decision-making approach.

We have designed the programme so that no one project carries all the intellectual weight of the programme putting the programme at risk should it fail. The breadth, nature and number of projects is one riskmitigating strategy-the whole is greater than the sum of its parts. Other strategies to ensure success including a formalized process for prioritization, peer-review, optimization of quality and monitoring project progress will be developed to ensure only the best, most relevant projects are advanced. Each project will be required to have a written proposal which will be reviewed by the Advisory Committee in terms of its relevance to the programme's objectives; potential to generate new knowledge, study design and methods; potential to achieve the intended outcomes/impact; and resources required.

Monthly team teleconferences and one annual face-toface meeting will maintain team cohesiveness and momentum and facilitate knowledge sharing. Team meetings, along with the annual review of projects by the Advisory Committee, will identify challenges faced by 
projects and marshal the collective wisdom of the team and/or Advisory Committee to overcome them. The diversity of the team and the richness of its content and scientific and knowledge user expertise will be a considerable asset for finding mitigating strategies.

\section{Research programme limitations}

The most significant limitation relates to the initial use of observational and quasi-experimental study designs. Given the focus on research partnerships, we expect that researchers and knowledge users will not be sympathetic or agreeable to experimental study designs that would require being randomized to use an IKT approach. However, to maximize overall scientific rigor, the research programme will rely on mixed methods and triangulation of findings and strive to select the most rigorous study designs for individual projects. For example, the use of both retrospective and prospective case studies is preferable to using only retrospective case studies. Another example is that we will be studying the influence of funded IKT studies by comparing the resulting impacts with the impacts of curiosity-driven research (essentially a non-randomized control group). We also anticipate that rigor will be increased by including projects that involve different types of knowledge users (e.g. patients, indigenous groups, clinicians, health services decision-makers, funders, etc.) and examine different levels of partnership (e.g. project, health authority, etc.). These settings will allow us to describe dominant patterns across varied arrangements, thereby enhancing the generalisability of the work. During the course of the 7year lifespan of the programme, we also expect to build on the lessons learned from the first wave of studies and propose and conduct more rigorous and methodologically innovate projects in subsequent waves. We also anticipate that in future prospective case studies, we will be able to introduce and evaluate interventions to improve partnering.

\section{Knowledge translation}

Our KT strategies consist of two approaches: IKT and end-of-project $\mathrm{KT} / \mathrm{knowledge}$ mobilization. In keeping with the focus on integrated knowledge translation, the Integrated Knowledge Translation Research Network will use an IKT approach in all of its studies to ensure the projects address the issues of concern of our knowledge user partners and hopefully produce useful findings that can be acted upon by our and other knowledge users.

Our end of project KT activities will be guided by the CIHR's Guide to Knowledge Translation Planning [69]. For academic audiences, we will produce peer-reviewed journal articles for relevant journals. For knowledge user and stakeholder audiences, we will use a number of strategies to disseminate our work. To facilitate dissemination, we will create a website for the network that will house all the tools and products we produce. We will create a web blog that will serve as a vehicle for early dissemination of findings, engaging the public and crossfertilizing our ideas with each other and scientists in other areas. We will use social media (e.g. Twitter) to create a presence of the IKT Research Network. We will also use a newsletter to inform audiences about our activities and to disseminate findings.

The suite of training materials, tools and sessions described above will be available online to help researchers and knowledge users build their capacity to engage in IKT. Another Network KT strategy for disseminating findings and capacity building in IKT will be the hosting of a bi-annual symposium on the State of the Art and Science of IKT. The symposium may occur around an annual meeting of the Canadian Association of Health Services and Policy Research (CAHSPR), KT Canada's annual general meeting or another conference. The purpose of these symposia will be to create a forum for knowledge users and researchers to share their experiences with partnering, present findings from the latest research on how best to undertake collaborative research, explore opportunities for working together/ network development and offer skill-building seminars and workshops on doing IKT, strategies for effective research partnerships and maintaining relationships.

We also intend to host events similar to the CIHR Best Brains Exchanges [70] with the National Alliance for Provincial Health Research Organizations (NAPHRO), the Health Charities Coalition of Canada, health sector organizations and the Canadian research Tri-Councils (Canadian Institutes of Health Research, Social Sciences and Humanities Research Council, National Science and Engineering Research Council) around the research programme findings. These exchanges will bring together researchers and policy makers/administrators in a relaxed and confidential environment to discuss IKT research and its policy implications. We will include trainees in these events so they can learn how they work, how to host them and to make connections with policy makers, health system managers and funders.

\section{Discussion}

We have proposed the first interdisciplinary, systematic and programmatic research endeavor and network focusing on IKT. The research programme was developed and will be executed with knowledge user organization executives, managers, policy makers, clinicians and patients. We will ground the programme in knowledge generated through systematic, scoping and realist reviews. Taking advantage of our pre-existing productive relationships with provincial, national and 
international organizations, we will use ongoing and future natural IKT experiments as multiple case studies in order to study IKT in depth. Case studies will be retrospective and prospective as the 7-year grant timeline will enable us to undertake prospective longitudinal studies of IKT. We will study, in real time, the initiation of partnerships, funding processes, the research lifecycle and then outcomes/impact post project. In the latter years of the programme, we anticipate that these living laboratories [71] will also facilitate testing of strategies to improve the efficiency and effectiveness of the IKT approach. The research will also provide scientific evidence on how to reliably and validly measure collaborative research partnerships and their impacts. Built into the programme is a vibrant training and mentoring environment for trainees and researchers interested in the science of IKT and its application.

By conducting a meta-synthesis of multiple case studies and other strategic studies undertaken during the early years of the programme, we will be able to demonstrate how IKT works, under which circumstances and with which knowledge user groups. We will determine what IKT can and cannot do and learn how researchers and knowledge users develop and maintain research partnerships. When available, we will assess the impact of IKT on health system and patient outcomes. We will also ascertain how to promote IKT among knowledge users/knowledge user organizations and researchers. Significant potential and timely opportunities exist for improving how IKT is practiced and supported. By better understanding IKT, developing instruments to measure it and its impact, and designing effective strategies that support IKT, we will be positioned to improve knowledge translation and more thoroughly reap the benefits of research.

\section{Abbreviations \\ AC: Advisory Committee; CIHR: Canadian Institutes of Health Research; CLARHC: Collaborations for Leadership in Applied Health Research and Care; CV: Curriculum vitae; FCR: Framework for collaborative research; HQP: Highly qualified personnel; IKT: Integrated knowledge translation; KT: Knowledge translation; KTA: Knowledge to action; RIC: Research Impact Continuum}

\footnotetext{
Acknowledgements

We would like to thank Danielle Rolfe for assisting with the drafting and editing of the manuscript.

lan Graham* is the Programme leader/PI and Scientific Director of the IKT Research Network. Current members of network are IKT experts: Gonzalo Alvarez*, Davina Banner* ${ }^{*}$, Mari Botti, Tracey Bucknall $\wedge$, Ingrid Botting* ${ }^{*}$, Julie Considine, Maxine Duke, Sandra Dunn*^, Trisha Dunning, Anna Gagliardi*, Heather Gainforth^, Wendy Gifford*^, Karen Harlos, Tanya Horsley $^{*} \wedge$, Alison Hutchinson^^, Monika Kastner*, Anita Kothari* ${ }^{*}$, Sara Kreindler* Linda Li, Martha MacLeod* ${ }^{*}$, Elizabeth Manias, Chris McCutcheon^, Jonathan Mitchell*, Theresa Montini*, Smita Pakhale, Mark Pearson, Bodil Rasmussen, Jo Rycroft-Malone* ${ }^{*}$, Nancy Salbach, Jonathan Salsberg*, Shannon Sibbald^ ${ }^{*}$ Kathryn Sibley, Dawn Stacey ${ }^{*} \wedge$, Harriette Van Spall, Margaret Watson*, Anna Williamson, David K Wright^, Euson Yeung^, Emily Jenkins.

Trainees: Sayna Bahrani, Fraser Bell*, Laura Boland, Clayon Hamilton, Janet Jull^, Jesse Leese, Graham MacDonald, Robert KD McLean^, Kelly Mrklas*^,
}

Tram Nguyen*^, Katrina Plamondon^, Danielle Rolfe, Katherine Salter*, Brianne Wood*, llja Ormel and Maria Zych.

Knowledge user experts: Donna Angus^, Beth Beaupre, Krista Connell*, Jeanette Edwards*, Shannon Fenton*, Michelle Gagnon^ , Kathryn Graham, Mary Elizabeth Harriman, Michael Hillmer*, Bev Holmes*^, Russell Ives*, lan Jones*, Karen Lee*, Ainslie Mihalchuk*, Michelle McEvoy*, Wendy Nicklin*, Mary Ann O'Brien, Patrick Odnokon*, Sheldon Permack*, Shannon Rogalski*, Hélène Sabourin*, Yves Savoie*, Gayle Scarrow*, Robert Sheldon*, Ann Sprague*, Anthony Tang*, Cathy Ulrich*, Pam Valentine*, Christina Weise*, George Wells*, Brock Wright*.

Knowledge translation and implementation science experts: Jamie Brehaut* Melissa Brouwers, Christina Godfrey^, Jeremy Grimshaw*, John Lavis*, France Legare*, David Moher*, Justin Presseau, Janet Squires*, Sharon Straus*, Jimmy Volmink.

Advisory Committee: Susan Law^, Anne Lyddiatt, Jacqueline Tetroe. $*=$ co-applicants on foundation grant, $\wedge=$ project lead

\section{Funding}

The study entitled: Moving knowledge into action for more effective practice, programs and policy: A research program focusing on integrated knowledge translation (known as the Integrated Knowledge Translation Research Network) is funded as part of a Canadian Institutes of Health Research, Foundation Grant Scheme: grant reference number FDN \#143237. Network projects $8 \mathrm{c}$ (Conducting patient-oriented research: an online tutorial for the use of a collaborative framework for community-research partnerships (PI: J Jull and ID Graham)) and 8d (Experiences of patient-researcher partnerships in integrated knowledge translation: a study to develop an online training for patient-oriented research (PI: S Law, ID Graham)) have received funding from the Ontario, Strategy for Patient-Oriented Research (SPOR) Support Unit (OSSU).

The Canadian Institutes of Health Research does not and will not have any role in influencing the study design, data collection, analysis and interpretation of the data or writing of manuscripts.

\section{Availability of data and materials}

Request for access to the data and any material will be considered by the Executive Committee and the specific project co-investigators.

\section{Authors' contributions}

IDG, with members of the network who were co-applicants on the CIHR proposal, designed the grant proposal (represented by an asterisk in the "Acknowledgements" section above). IDG, Principal Investigator on the Foundation Grant and Scientific Director of the IKT Research Network, drafted the first version of the protocol manuscript on behalf of the network. Named co-authors, who are project leads, led the design of their projects. All authors (lan D. Graham, Anita Kothari, Chris McCutcheon, Donna Angus, Davina Banner, Tracey Bucknall, Sandra Dunn, Michelle Gagnon, Wendy Gifford, Christina Godfrey, Bev Holmes, Tanya Horsley, Alison M. Hutchinson, Janet Jull, Susan Law, Martha MacLeod, Robert K.D. McLean, Kelly Mrklas, Tram Nguyen, Katrina Plamondon, Jo Rycroft-Malone, Shannon L Sibbald, Kathryn M. Sibley, Dawn Stacey, David Kenneth Wright, Euson Yeung) reviewed, critically revised and approved the final version of the manuscript.

\section{Ethics approval and consent to participate}

Each individual project involving human participants will obtain Research Ethics Approval from the appropriate Research Ethics Board(s). Project 1a (Comparing and contrasting integrated knowledge translation with five approaches often used in knowledge translation: Identifying and synthesizing germinal literature and consulting with experts- PI: Tram Nguyen, lan Graham) has ethics approval from the Ottawa Health Sciences Network Research Ethics Board (OHSN- REB \#2017038-01H). Project 2a (An evaluation of an academic-health services partnership network) has ethics approval from the Deakin University Human Ethics Advisory Group-Health (HEAG-H \#132-2015).

\section{Consent for publication}

Not applicable.

\section{Competing interests}

Network members AMH, JP, JS and SS are Associate Editors of Implementation Science, and MB, IDG, JG, BJH, FL and JR-M are on the editorial board of 
Implementation Science. All decisions about this manuscript will be made by another editor. JRM is Director of the National Institute for Health Research's Health

Services and Development Research Funding Programme. The authors declare that they have no other competing interests. The views expressed are those of the authors and do not represent the views of any organization or agency to which they are affiliated.

\section{Publisher's Note}

Springer Nature remains neutral with regard to jurisdictional claims in published maps and institutional affiliations.

\section{Author details}

${ }^{1}$ School of Epidemiology and Public Health, University of Ottawa, 307D- 600 Peter Morand Crescent, Ottawa, ON K1G 5Z3, Canada. ${ }^{2}$ School of Health Studies, Western University, Health Sciences Building, Room 222, London, Ontario N6A 5B9, Canada. Integrated Knowledge Translation Research Network, Centre for Practice-Changing Research, The Ottawa Hospital Research Institute, 501 Smyth Road, Box 711, Ottawa, Ontario K1H 8L6, Canada.

Received: 5 October 2017 Accepted: 18 December 2017

Published online: 02 February 2018

\section{References}

1. Macleod MR, Michie S, Roberts I, Dirnagl U, Chalmers I, loannidis JPA, et al. Biomedical research: increasing value, reducing waste. Lancet. 2014;383: 101-4. https://doi.org/10.1016/S0140-6736(13)62329-6.

2. Tetroe JM, Graham ID, Foy R, Robinson N, Eccles MP, Wensing M, et al. Health research funding agencies' support and promotion of knowledge translation: an international study. Milbank Q. 2008;86:125-55. https://doi. org/10.1111/j.1468-0009.2007.00515.x

3. Kothari A, McCutcheon C, Graham ID; for the IKT Research Network. Defining integrated knowledge translation and moving forward: a response to recent commentaries. Kerman Univ Med Sci. 2017;6:299-300. https:/doi.org/10.15171/ijhpm.2017.15.

4. Graham ID, Tetroe JM, Maclean R. Some basics of integrated knowledge translation research. In: Graham ID, Tetroe JM, Pearson A, editors. Turning knowledge into action: practical guidance on how to do integrated knowledge translation research. Adelaide: Lippincott-JBI; 2014.

5. Bowen SJ, Graham ID. From knowledge translation to engaged scholarship: promoting research relevance and utilization. Arch Phys Med Rehabil. 2013; 94(1 SUPPL):S3-8. https://doi.org/10.1016/j.apmr.2012.04.037.

6. Denis J-L, Lomas J. Convergent evolution: the academic and policy roots of collaborative research. J Health Serv Res Policy. 2003;8(2_suppl):1-6. https:// doi.org/10.1258/135581903322405108.

7. Gibbons $M$. The new production of knowledge: the dynamics of science and research in contemporary societies. London: SAGE Publications; 1994.

8. Jagosh M, Macaulay A, Pluye P, Salsberg J, Bush P, HENDERSON J, et al. Uncovering the benefits of participatory research: implications of a realist review for health research and practice. Milbank Q. 2012;90:311-46. https:// doi.org/10.1111/j.1468-0009.2012.00665.x.

9. Macaulay AC, Ing A, Salsberg J, McGregor A, Saad-Haddad C, Rice J, et al. Community-based participatory research: lessons from sharing results with the community: Kahnawake Schools Diabetes Prevention Project. Prog Community Heal Partnerships Res Educ Action. 2007;1:143-52. https://doi. org/10.1353/cpr.2007.0010.

10. Salsberg J. Integrated knowledge translation and participatory research. In: Graham ID, Tetroe JM, Pearson A, editors. Turning knowledge into action: practical guidance on how to do integrated knowledge translation research. Adelaide, SA: Lippincott-JBI; 2014.

11. Kothari A, Wathen CN, McEachran M, Gauvin FP, Martin E, Mercer SL, et al. A critical second look at integrated knowledge translation. Health Policy. 2013; 109:187-91. https://doi.org/10.1016/j.healthpol.2012.11.004.

12. Holmes B, Scarrow G, Schellenberg M. Translating evidence into practice: the role of health research funders. Implement Sci. 2012;7:39. https://doi. org/10.1186/1748-5908-7-39.

13. Nasser M, Clarke M, Chalmers I, Brurberg KG, Nykvist H, Lund H, et al. What are funders doing to minimise waste in research? Lancet (London, England). 2017;389:1006-7. https://doi.org/10.1016/S0140-6736(17)30657-8.
14. Sibbald SL, Tetroe J, Graham ID. Research funder required research partnerships: a qualitative inquiry. Implement Sci. 2014;9:176. https://doi.org/ 10.1186/s13012-014-0176-y.

15. Canadian Institutes of Health Research. About us-CIHR. http://www.cihrirsc.gc.ca/e/29418.html\#5.2. Accessed 29 Sept 2017.

16. Canadian Institutes of Health Research. Industry-Partnered Collaborative Research. https://www.researchnet-recherchenet.ca/rnr16/vwOpprtntyDtls. do?prog $=1871 \&$ view $=$ currentOpps\&org $=$ CIHR\&type $=$ EXACT\&resultCount $=$ 25\&sort=program\&all=1\&masterList=true. Accessed 20 Sept 2017.

17. Canadian Institutes of Health Research. Partnerships for Health System Improvement (PHSI) —Application Instructions_CIHR. http://www.cihr-irsc. gc.ca/e/39315.html. Accessed 20 Sept 2017.

18. Canadian Institutes of Health Research. Strategy for Patient-Oriented Research-CIHR. http://www.cihr-irsc.gc.ca/e/41204.html. Accessed 20 Sept 2017.

19. CRIO: Collaborative Research and Innovation Opportunities. Alberta Innovates: Health Solutions. http://www.aihealthsolutions.ca/funding/healthresearch-funding/crio-collaborative-research-and-innovation-opportunities/. Accessed 20 Sept 2017.

20. Patient-Centered Outcomes Research Institute. PCORI_Patient-Centered Outcomes Research Institute. https://www.pcori.org/. Accessed 20 Sept 2017.

21. Quality Enhancement Research Initiative (QUERI). https://www.queri. research.va.gov/. Accessed 20 Sept 2017.

22. NIDRR Programs and Projects. 2007. https://www.acl.gov/about-acl/aboutnational-institute-disability-independent-living-and-rehabilitation-research. Accessed 22 Dec 2017

23. Academic Collaborative Centres Public Health-ZonMw. https://www zonmw.nl/en/research-and-results/prevention/programmas/programmedetail/academic-collaborative-centres-public-health/. Accessed 20 Sept 2017.

24. Fisk NM, Wesselingh SL, Beilby JJ, Glasgow NJ, Puddey IB, Robinson BG, et al. Academic health science centres in Australia: let's get competitive. Med J Aust 2011;194:59-60. http://www.ncbi.nlm.nih.gov/pubmed/21241215. Accessed 20 Sept 2017.

25. Department of Health. Best research for best health. A New Natl Heal Res Strateg NHS Contrib to Heal Res England. 2006;17(Suppl.1):11-315. https:// doi.org/10.1111/j.1471-1842.2009.00842.x.

26. Rycroft-Malone J. From knowing to doing-from the academy to practice comment on "the many meanings of evidence: implications for the translational science agenda in healthcare". Int J Heal Policy Manag. 2014;2: 45-6. https://doi.org/10.15171/ijhpm.2014.08.

27. Rycroft-Malone J, Burton CR, Bucknall T, Graham ID, Hutchinson AM, Stacey D. Collaboration and co-production of knowledge in healthcare: opportunities and challenges. Int J Heal Policy Manag. 2016;5:221-3. https:// doi.org/10.15171/ijhpm.2016.08.

28. Cooke J, Langley J, Wolstenholme D, Hampshaw S. "Seeing" the difference: the importance of visibility and action as a mark of "authenticity" in coproduction comment on "collaboration and co-production of knowledge in healthcare: opportunities and challenges". Int J Heal Policy Manag. 2016;6: 345-8. https://doi.org/10.15171/ijhpm.2016.136.

29. El-Jardali F, Fadlallah R. A call for a backward design to knowledge translation. Int J Heal Policy Manag. 2015;4:1-5. https://doi.org/10.15171/ ijhpm.2015.10.

30. Smith N, Cornelissen E, Mitton C. Reflecting on backward design for knowledge translation comment on "a call for a backward design to knowledge translation". Int J Heal Policy Manag. 2015;4:541-3. https://doi. org/10.15171/ijhpm.2015.92.

31. Bowen S, Graham ID. Backwards design or looking sideways? knowledge translation in the real world comment on "A call for a backward design to knowledge translation". Int J Heal Policy Manag. 2015;4:545-7. https://doi. org/10.15171/ijhpm.2015.71.

32. Mitton C, Adair CE, McKenzie E, Patten SB, Waye Perry B. Knowledge transfer and exchange: review and synthesis of the literature. Milbank Q. 2007;85: 729-68. https://doi.org/10.1111/j.1468-0009.2007.00506.X.

33. Gagliardi AR, Berta W, Kothari A, Boyko J, Urquhart R. Integrated knowledge translation (IKT) in health care: a scoping review. Implement Sci. 2015;11:38. https://doi.org/10.1186/s13012-016-0399-1.

34. Tricco AC, Zarin W, Rios P, Pham B, Straus SE, Langlois EV. Barriers, facilitators, strategies and outcomes to engaging policymakers, healthcare managers and policy analysts in knowledge synthesis: a scoping review protocol. BMJ Open. 2016;6:e013929. https://doi.org/10.1136/bmjopen-2016013929. 
35. Camden C, Shikako-Thomas K, Nguyen T, Graham E, Thomas A, Sprung J, et al. Engaging stakeholders in rehabilitation research: a scoping review of strategies used in partnerships and evaluation of impacts. Disabil Rehabil. 2015:37:1390-400. https://doi.org/10.3109/09638288.2014.963705.

36. Cargo M, Mercer SL. The value and challenges of participatory research: strengthening its practice. Annu Rev Public Health. 2008;29:325-50. https:// doi.org/10.1146/annurev.publhealth.29.091307.083824.

37. D'Andreta D, Scarbrough $H$, Evans S. The enactment of knowledge translation: a study of the collaborations for leadership in applied health research and care initiative within the English National Health Service. J Health Serv Res Policy. 2013;18(3_suppl):40-52. https://doi.org/10.1177/ 1355819613499902.

38. Rycroft-Malone J, Wilkinson J, Burton CR, Harvey G, McCormack B, Graham I, et al. Collaborative action around implementation in collaborations for leadership in applied health research and care: towards a programme theory. J Health Serv Res Policy. 2013;18(3_suppl):13-26. https://doi.org/10. $1177 / 1355819613498859$

39. Soper B, Yaqub O, Hinrichs S, Marjanovich S, Drabble S, Hanney S, et al. CLAHRCs in practice: combined knowledge transfer and exchange strategies, cultural change, and experimentation. J Health Serv Res Policy. 2013;18(3_suppl):53-64. https://doi.org/10.1177/1355819613499903.

40. Walshe K, Davies HT. Health research, development and innovation in England from 1988 to 2013: from research production to knowledge mobilization. J Health Serv Res Policy. 2013;18(3_suppl):1-12. https://doi.org/ $10.1177 / 1355819613502011$.

41. Martin GP, McNicol S, Chew S. Towards a new paradigm in health research and practice? J Health Organ Manag. 2013;27:193-208. https://doi.org/10. 1108/14777261311321770.

42. Rycroft-Malone J, Burton CR, Wilkinson J, Harvey G, McCormack B, Baker R, et al. Collective action for implementation: a realist evaluation of organisational collaboration in healthcare. Implement Sci. 2016;11:17. https://doi.org/10.1186/s13012-016-0380-z.

43. McLean R, Tucker J. Evaluation of CIHR's knowledge translation funding program evaluation report 2013. 2013. http://cihr-irsc.gc.ca/e/documents/ kt_evaluation_report-en.pdf. Accessed 22 Sept 2017.

44. McLean RK, Graham ID, Bosompra K, Choudhry Y, Coen SE, Macleod M, Manuel C, McCarthy R, Mota A, Peckham D, Tetroe JM, Tucker J. Understanding the performance and impact of public knowledge translation funding interventions: protocol for an evaluation of Canadian Institutes of Health Research knowledge translation funding programs. Implement Sci. 2012;7:57. https://doi.org/10.1186/1748-5908-7-57.

45. Bowen S, Botting I, Graham ID, Huebner L-A. Beyond "two cultures": guidance for establishing effective researcher/health system partnerships. Int J Heal policy Manag. 2016;6:27-42. https://doi.org/10. 15171/ijhpm.2016.71.

46. Tantchou Dipankui M. Collaboration between researchers and knowledge users in health technology assessment: a qualitative exploratory study. Int J Heal policy Manag. 2016;6:437-46. https://doi.org/10.15171/ijhpm.2016.153.

47. Gagliardi AR, Dobrow MJ. Identifying the conditions needed for integrated knowledge translation (IKT) in health care organizations: qualitative interviews with researchers and research users. BMC Health Serv Res. 2016; 16:256. https://doi.org/10.1186/s12913-016-1533-0.

48. Gainforth $H L$, Latimer-Cheung AE, Athanasopoulos P, Moore S, Ginis KAM. The role of interpersonal communication in the process of knowledge mobilization within a community-based organization: a network analysis. Implement Sci. 2014;9:59. https://doi.org/10.1186/1748-5908-9-59.

49. Fleurence $R L$, Forsythe $L P$, Lauer $M$, Rotter J, loannidis JPA, Beal A, et al. Engaging patients and stakeholders in research proposal review: the Patient-Centered Outcomes Research Institute. Ann Intern Med. 2014;161: 122. https://doi.org/10.7326/M13-2412.

50. Zittleman L, Wright L, Ortiz BC, Fleming C, Loudhawk-Hedgepeth C, Marshall J, et al. Colorado immersion training in community engagement: because you can't study what you don't know. Prog Community Heal Partnerships Res Educ Action. 2014;8:117-24. https://doi.org/10.1353/cpr. 2014.0006

51. Adily A, Black D, Graham ID, Ward JE. Research engagement and outcomes in public health and health services research in Australia. Aust N Z J Public Health. 2009;33:258-61. https://doi.org/10.1111/j.1753-6405.2009.00385.x.

52. Sibley KM, Roche PL, Bell CP, Temple B, Wittmeier KDM. A descriptive qualitative examination of knowledge translation practice among health researchers in Manitoba, Canada. BMC Health Serv Res. 2017;17:627. https:// doi.org/10.1186/s12913-017-2573-9.

53. Gagliardi AR, Kothari A, Graham ID. Research agenda for integrated knowledge translation (IKT) in healthcare: what we know and do not yet know. J Epidemiol Community Health. 2017;71:105-6. https://doi.org/10. 1136/jech-2016-207743.

54. Graham ID. A proposed research translation strategy 2012-2015. Adelaide: South Australian Health and Medical Research Institute; 2012.

55. Graham ID, Logan J, Harrison MB, Straus SE, Tetroe J, Caswell W, et al. Lost in knowledge translation: time for a map? J Contin Educ Heal Prof. 2006;26: 13-24. https://doi.org/10.1002/chp.47.

56. Straus SE, Tetroe J, Graham ID. Knowledge translation in health care: moving from evidence to practice. Chichester: Wiley; 2013.

57. Gifford W. W, Graham I, Eldh AC, Lefebre N. Theoretical foundations of dissemination and implementation leadership: a conceptual model for leadership development. In: The 7th Annual Conference on the Science of Dissemination and Implementation, Bethesda; 2014. http://www.diva-portal. org/smash/record.jsf?pid=diva2\%3A848744\&dswid=sno. Accessed 20 Sept 2017.

58. Gifford WA, Davies BL, Graham ID, Tourangeau A, Woodend AK, Lefebre N. Developing leadership capacity for guideline use: a pilot cluster randomized control trial. Worldviews Evidence-Based Nurs. 2013;10:51-65. https://doi. org/10.1111/j.1741-6787.2012.00254.x.

59. Pawson R, Greenhalgh T, Harvey G, Walshe K. Realist review-a new method of systematic review designed for complex policy interventions. J Health Serv Res Policy. 2005;10(1_suppl):21-34. https://doi.org/10.1258/ 1355819054308530

60. Levac D, Colquhoun H, O'Brien KK. Scoping studies: advancing the methodology. Implement Sci. 2010;5:69. https://doi.org/10.1186/1748-59085-69.

61. Arksey H, O'Malley L. Scoping studies: towards a methodological framework. Int J Soc Res Methodol. 2005;8:19-32. https://doi.org/10.1080/ 1364557032000119616.

62. Yin RK. Case study research: design and methods. Fifth. SAGE: Thousand Oaks.

63. Stake RE. Multiple case study analysis. New York: Guilford Press; 2006. https://www.guilford.com/books/Multiple-Case-Study-Analysis/Robert-Stake/ 9781593852481/reviews. Accessed 25 Sept 2017.

64. Creswell JW, Plano Clark VL. Designing and conducting mixed methods research. Second. Thousand Oaks: SAGE Publications; 2011.

65. Donnelly F, Wiechula R. Clinical placement and case study methodology: a complex affair. Nurse Educ Today. 2012;32:873-7. https://doi.org/10.1016/j. nedt.2012.02.010.

66. Pettigrew A. Leading organisational change: Frameworks and findings from Warwick research 1985-2002. Report for the Office of Public Sector Reform. London: Cabinet Office; 2002.

67. Kothari A, Sibbald SL, Wathen CN. Evaluation of partnerships in a transnational family violence prevention network using an integrated knowledge translation and exchange model: a mixed methods study. Heal Res Policy Syst. 2014;12:25. https://doi.org/10.1186/1478-4505-12-25.

68. Kothari A, MacLean L, Edwards N, Hobbs A. Indicators at the interface: managing policymaker-researcher collaboration. Knowl Manag Res Pract. 2011;9:203-14. https://doi.org/10.1057/kmrp.2011.16

69. Canadian Institutes of Health Research. Guide to knowledge translation planning at CIHR: integrated and end-of-grant approaches. Ottawa; 2012. http://www.cihr-irsc.gc.ca/e/45321.html.

70. Canadian Institutes of Health Research. Best Brains Exchanges. http://www. cihr-irsc.gc.ca/e/43978.html. Accessed 29 Jan 2018.

71. Armbruster RR, Begun JW, Duncan AK. An in-house learning laboratory for patient-centered innovation. J Healthc Qual. 31:10-17. http://www.ncbi.nlm. nih.gov/pubmed/19343896. Accessed 20 Sept 2017. 